\title{
Pachychoroid neovasculopathy in extramacular choroidal neovascularization
}

\author{
This article was published in the following Dove Press journal: \\ Clinical Ophthalmology \\ 12 July 2016 \\ Number of times this article has been viewed
}

\section{Mrinali Patel Gupta \\ Irene Rusu \\ Carly Seidman \\ Anton Orlin \\ Donald J D'Amico \\ Szilard Kiss}

Department of Ophthalmology, Weill Cornell Medicine, New YorkPresbyterian Hospital, New York, NY, USA
Correspondence: Szilard Kiss Weill Cornell Medicine Eye Associates Retina Division, I Ith Floor, I 305 York Ave, New York, NY I002I, USA

Tel +l 6469622020

Fax + I 6469620600

Emailszk700I@med.cornell.edu
Purpose: To review a series of extramacular choroidal neovascular membranes (CNVMs) in the context of their choroidal features, as determined by optical coherence tomography (OCT).

Methods: Patients with extramacular CNVMs were identified from a tertiary care center through a review of records. The charts and cases were reviewed using multimodal imaging including fundus photography, OCT, fluorescein angiography (FA), and indocyanine angiography (ICG).

Results: Of six patients with extramacular CNVMs evaluated in this series, four patients (66.7\%) exhibited pachychoroidopathy on OCT imaging under or adjacent to the extramacular CNVM. All four of these patients also exhibited pachychoroidopathy in the macular OCT distant from the CNVM.

Conclusion: Pachychoroidopathy is implicated in some cases of extramacular CNVMs. This represents the first report, to our knowledge, of pachychoroidopathy in extramacular CNVM.

Keywords: choroidal neovascularization, pachychoroidopathy, pachychoroid neovasculopathy, peripheral disciform lesions, extramacular choroidal neovascularization, polypoidal choroidal vasculopathy

\section{Introduction}

"Pachychoroidopathy" is a recently coined term to describe the shared choroidal features of thickened choroid and dilated outer choroidal vessels that link a spectrum of retinal conditions including pigmentary change, central serous chorioretinopathy (CSCR), some cases of type 1 (sub-retinal pigment epithelium [RPE]) choroidal neovascularization $(\mathrm{CNV})$, and polypoidal choroidal vasculopathy (PCV). ${ }^{1-3}$ Although controversy remains regarding pachychoroidopathy, recent studies suggest that the genetics and pathogenesis of pachychoroidopathy, as well as response to therapies, may be distinct from that seen in related conditions such as age-related macular degeneration (AMD). Previously, pachychoroidopathy-related type $1 \mathrm{CNV}$, termed "pachychoroid neovasculopathy," has been described in patients with choroidal neovascular membranes (CNVMs) localized to the central macula. To evaluate the role of pachychoroidopathy in extramacular CNVMs, also known as peripheral disciform lesions, we retrospectively reviewed a series of cases of extramacular CNVMs in the context of their associated choroidal findings.

\section{Methods}

We retrospectively analyzed a nonconsecutive case series of six patients with extramacular CNVMs. All patients were examined by the Retina Service of the Department of Ophthalmology at Weill Cornell Medicine, New York, NY, USA, with slit 
lamp biomicroscopy, indirect ophthalmoscopy, color fundus photography (Optos ${ }^{\mathrm{TM}}$ Panoramic 200A; Optos PLC, Dunfermline, UK), and enhanced-depth imaging optical coherence tomography (EDI-OCT; Heidelberg Spectralis; Heidelberg Engineering, Inc, Vista, CA, USA). EDI-OCT was performed through the macula in all cases, as well as through the CNVM lesion itself whenever possible. Fluorescein angiography (FA) and indocyanine green angiography (ICG) were also reviewed, when available. The study period was January 2015 to December 2015. This study was approved by the Institutional Review Board of Weill Cornell Medicine and was performed in a Health Care Portability and Accountability Act compliant manner. No informed consent was obtained as this was a retrospective case review study.

\section{Results}

The six cases reported had an average age of 74.8 years (range 56 to 87 years). Five were female and one was male. Average follow-up was 20 months (range 2 to 35 months). The clinical and choroidal features of the six cases are summarized in Table 1.

Of the six cases reviewed, four (66.7\%; cases $1-4)$ exhibited pachychoroidopathy (Figures 1-4). These patients were all females ranging in age from 56 to 84 years. Two of these patients presented with photopsia (cases 1 and 3). All four patients exhibited an extramacular CNVM, with macular EDI-OCT revealing thick choroid with dilated outer choroidal vessels. EDI-OCT obtained through the CNVM itself (cases 1-3) revealed Type 1 CNVM overlying thick choroid with dilated outer choroidal vessels which were inwardly displaced and abutting the RPE. EDI-OCT was not possible through the large hemorrhagic pigment epithelial detachment (PED) in case 4, but OCT in the retina adjacent to this lesion similarly revealed thick choroid and dilated outer choroidal vessels. FA was performed in each case and revealed leakage consistent with CNVM in three patients (cases 1-3); case 4 exhibited blockage by the large sub-RPE hemorrhage. ICG was performed in only one case (case 2) and revealed polypoidal neovascular lesions in the area of extramacular CNVM, as well as a few small polypoid lesions in the fellow eye (Figure 2C and D). Two patients (cases 1 and 3) were treated with intravitreal anti-vascular endothelial growth factor (VEGF) injections due to increased exudation or symptomatic CNVM. Case 1 noted no significant response to anti-VEGF therapy nor of her photopsia, while case 3 exhibited stable exudation but resolution of photopsia after therapy. All four cases maintained stable visual acuity at last follow-up.
Case 1 is a representative case of extramacular pachychoroid neovasculopathy. This patient is a 79 year old Caucasian female who presented with symptoms of photopsia OD (oculus dexter [right eye]). Visual acuity (VA) was 20/15. There were rare macular RPE changes and subtle choroidal folds consistent with increased choroidal thickness, as well as a superonasal peripapillary CNVM with associated exudation OD (Figure 1A). EDI-OCT of the macula revealed thick choroid with subtle choroidal folds overlying dilated outer choroidal vessels OD (Figure 1B). EDI-OCT through the superonasal exudative lesion OD revealed thickened choroid and dilated outer choroidal vessels with overlying RPE disturbance and type 1 neovascularization (Figure 1C). The dilated choroidal vessels were inwardly displaced and abutted the RPE. Ultrawide-field FA revealed leakage from an occult choroidal neovascular membrane (Figure 1D and E). An ICG was not performed. The lesion was observed, but increased exudation and symptoms were noted 11 months later, prompting treatment with intravitreal ranibizumab (Lucentis ${ }^{\circledR}$; Hoffman-La Roche Ltd., Basel, Switzerland) 0.5 mg OD and subsequently, aflibercept 2 mg (EYLEA, Regeneron Pharmaceuticals, Inc., Tarrytown, NY, USA) twice, with no significant improvement. At the most recent follow-up, 35 months after initial presentation, exudation was noted to have further increased (Figure 1F), though the macula remains spared and $\mathrm{VA}$ is $20 / 20$. The patient declined further intervention such as anti-VEGF therapy or photodynamic therapy.

Two of the six cases (cases 5-6) exhibited extramacular CNVM without pachychoroidopathy (Figures 5 and 6). Both were male patients in their 80 s who presented with macular drusen and RPE changes and incidentally noted extramacular CNVM with hemorrhage in the far temporal periphery. EDI-OCT through the macula revealed RPE changes and markedly thinned choroid. The lesions were too peripheral to perform EDI-OCT through the CNVM itself.

\section{Discussion}

Studies have recently described "pachychoroidopathy", as a spectrum of conditions (including pigmentary changes, CSCR, some cases of type 1 neovascularization, and polypoidal choroidal vasculopathy) that share common features of thickened choroid and dilated outer choroidal vessels. ${ }^{1-3}$ On the milder side of this spectrum is pachychoroid pigment epitheliopathy (PPE), in which choroidal thickening and dilated outer choroidal vessels are associated with overlying RPE disturbances. PPE may be a forme fruste of CSCR, sharing the underlying choroidal pathology but lacking the classic macular subretinal fluid or gravitational tracks 


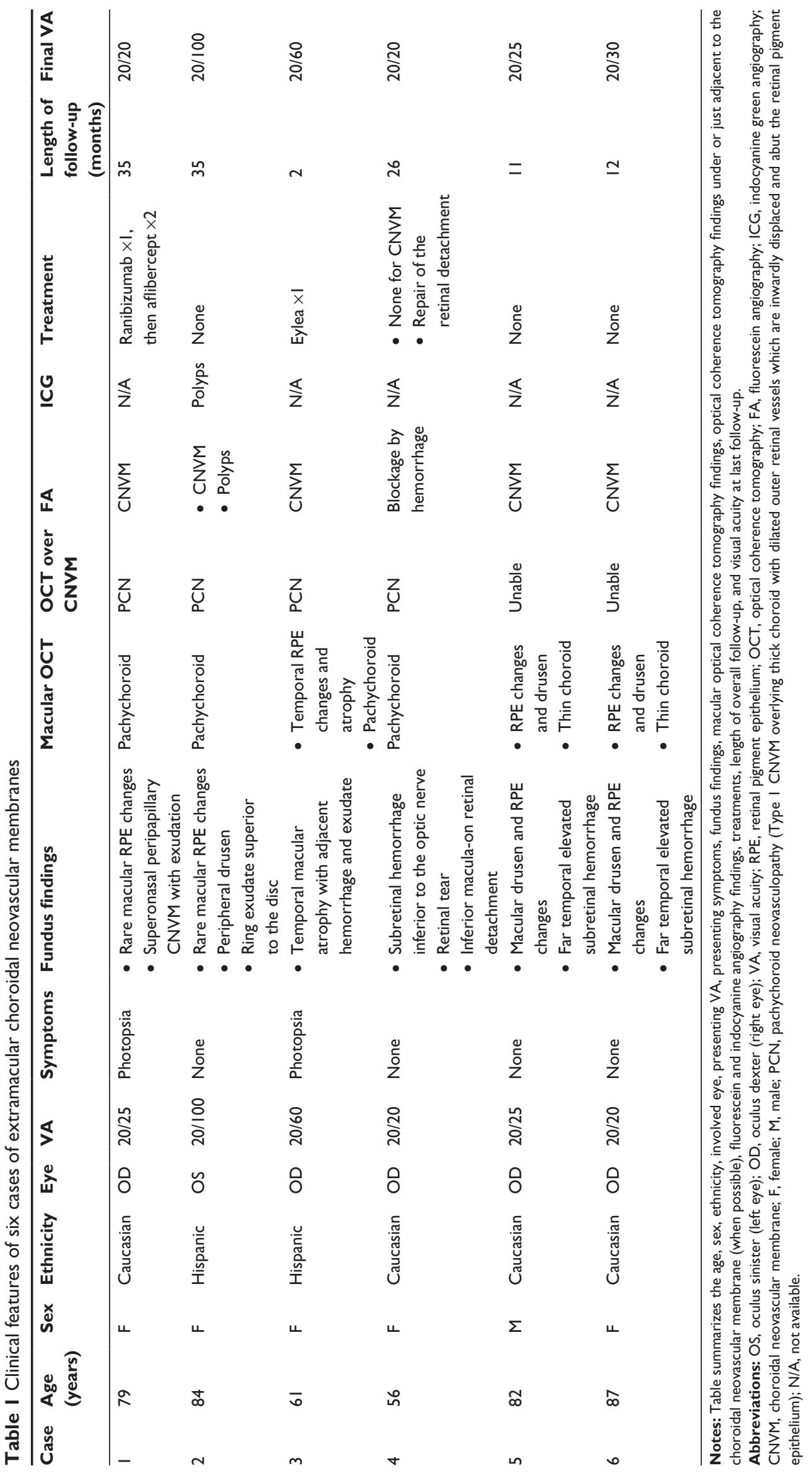


A
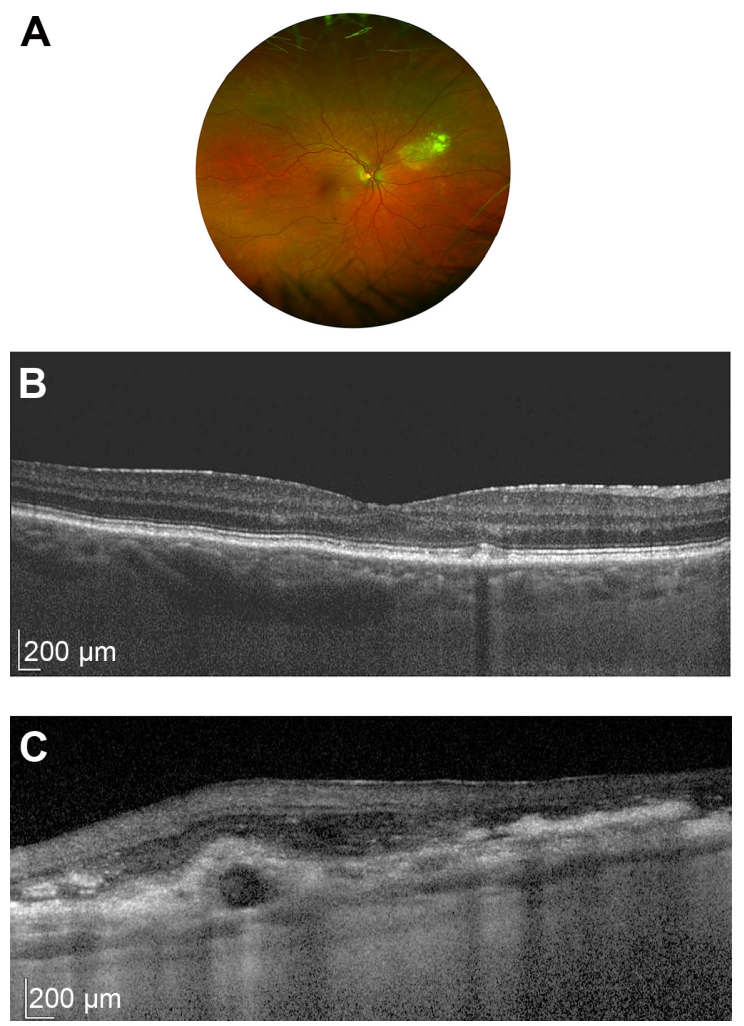

D

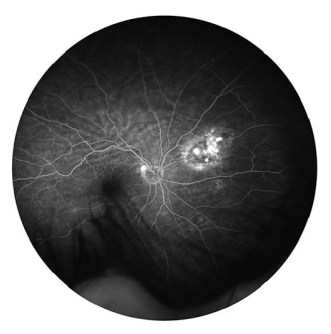

E

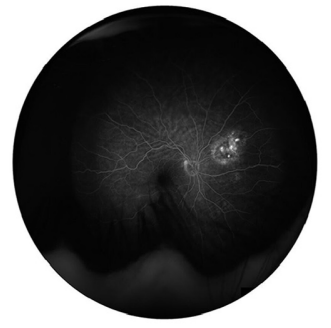

$\mathbf{F}$

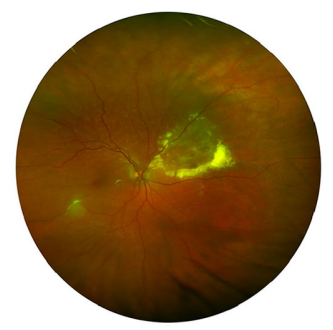

Figure I Case I ocular imaging.

Notes: Color fundus photograph OD for case I reveals rare macular RPE changes and a superonasal peripapillary CNVM with exudation (A). EDI-OCT (B) of the macula OD reveals rare drusen and choroidal folds overlying thickened choroid with dilated choroidal vessels. EDI-OCT through the superonasal CNVM (C) reveals Type I CNVM overlying thickened choroid and dilated outer choroidal vessels. The dilated choroidal vessels are inwardly displaced and abutting the RPE. FA (D) I minute, 7 seconds; (E) 2 minutes, 53 seconds, reveals leakage suggestive of occult CNVM. Color fundus photo (F) revealed further increase in exudation, despite treatment with multiple anti-VEGF agents. Abbreviations: OD, oculus dexter (right eye); CNVM, choroidal neovascular membrane; EDI-OCT, enhanced-depth imaging optical coherence tomography; OCT, optical coherence tomography; RPE, retinal pigment epithelium; FA, fluorescein angiography; VEGF, vascular endothelial growth factor.

of CSCR. ${ }^{3}$ In some patients with PPE, longstanding RPE changes and possibly RPE detachment can lead to disruption of Bruch's membrane, leading to ingrowth of sub-RPE choroidal neovascular membranes, resulting in Type $1 \mathrm{CNV}$. These neovascular membranes overlie areas of thickened choroid with dilated outer choroidal vessels and have been termed "pachychoroid neovasculopathy." Over time these neovascular membranes can evolve into branching vascular networks with polypoid lesions seen in PCV. Prior studies have demonstrated associations between CSCR and Type 1 neovascularization, longstanding Type 1 neovascularization and $\mathrm{PCV}^{4-7}$ and CSCR and PCV. ${ }^{8-12}$ The newly conceived pachychoroidopathy suggests that a pathologic choroidal process characterized by dilated outer choroidal vessels and thick choroid is the underlying common denominator.

Thus far, pachychoroid neovasculopathy has been reported in patients with CNVMs in the central macula., There are no reports of pachychoroid neovasculopathy in cases with extramacular CNVMs. To evaluate whether pachychoroidopathy may be implicated in extramacular
CNVMs, we retrospectively evaluated choroidal features in the central macula and, when possible, under the CNVMs, in a series of six extramacular CNVM cases evaluated at Weill Cornell Medical College. This series is the first, to our knowledge, to examine these peripheral CNVMs in the context of their choroidal findings.

In this small retrospective case series, four of six cases of extramacular CNVMs exhibited pachychoroidopathy - ie, thickened choroids and dilated outer choroidal vessels under or adjacent to the CNVM. This represents the first report of pachychoroid neovasculopathy outside of the central macula. Another feature of pachychoroid neovasculopathy is inward displacement of the dilated outer choroidal vessels below the lesions, such that they are abutting the RPE. This was noted in all four cases. Two patients with pachychoroidopathy presented with symptoms of photopsia. Whether this symptomatology is related to the underlying condition is unclear. In all four cases demonstrating pachychoroidopathy under or adjacent to the extramacular CNVM, the macular OCT also 
A
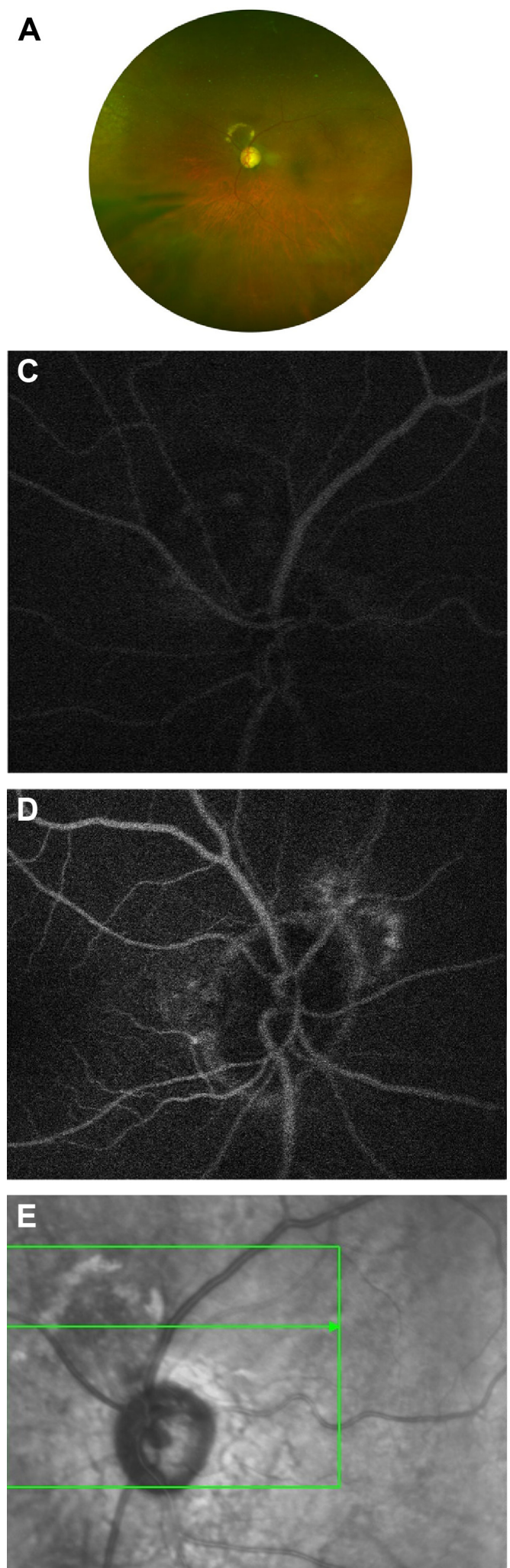
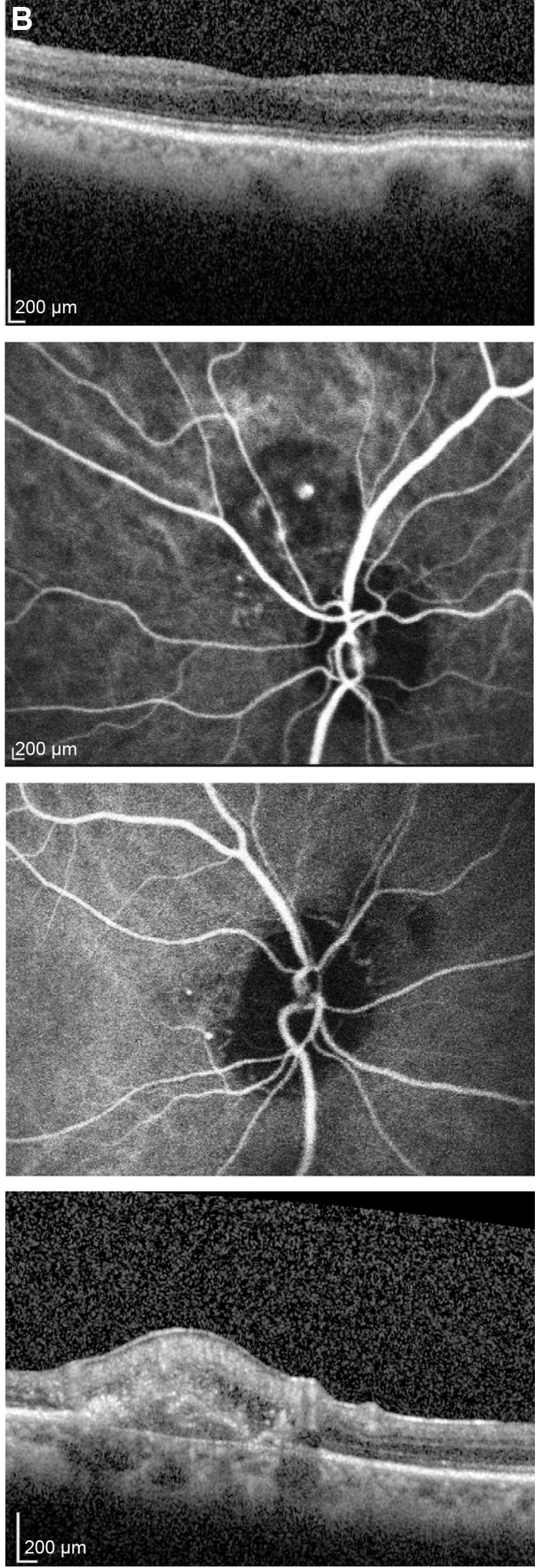

Figure 2 Case 2 ocular imaging.

Notes: Color fundus photograph OS for case 2 reveals rare macular RPE changes, peripheral drusen, and a ring exudate superior to the disc (A). EDI-OCT of the macula OS reveals choroidal folds overlying thickened choroid with dilated outer choroidal vessels (B). FA (C, left panel) and ICG (C, right panel) reveal polypoidal CNVM in the area of exudation OS (C: I minute, I second), as well as polypoidal lesions in the peripapillary region OD (D: 3 minutes, 53 seconds; left panel, FA; right panel, ICG). EDI-OCT (E, right panel) through the CNVM OS (E, left panel; green arrow signifying location of corresponding EDI-OCT image) reveals thickened choroid and dilated outer choroidal vessels that are inwardly displaced and abutting the RPE.

Abbreviations: OS, oculus sinister (left eye); OD, oculus dexter (right eye); RPE, retinal pigment epithelium; EDI-OCT, enhanced-depth imaging optical coherence tomography; FA, fluorescein angiography; ICG, indocyanine green angiography; CNVM, choroidal neovascular membrane. 

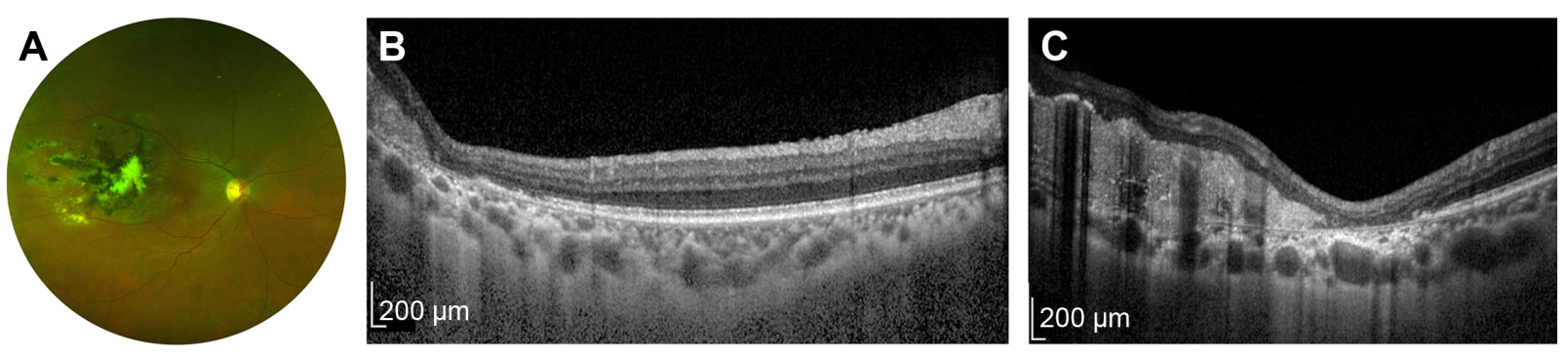

Figure 3 Case 3 ocular imaging.

Notes: Color fundus photograph OD for case 3 reveals temporal macular RPE changes and atrophy with an area of hemorrhage at the inferior border of the scar with associated exudation (A). EDI-OCT through the macula OD reveals temporal macular RPE changes and atrophy with underlying thickened choroid and dilated outer choroidal vessels (B). EDI-OCT through CNVM itself $(\mathbf{C})$ reveals thick choroid and markedly dilated outer choroidal vessels which are inwardly displaced and abutting the RPE. Abbreviations: OD, oculus dexter (right eye); RPE, retinal pigment epithelium; EDI-OCT, enhanced-depth imaging optical coherence tomography; CNVM, choroidal neovascular membrane.
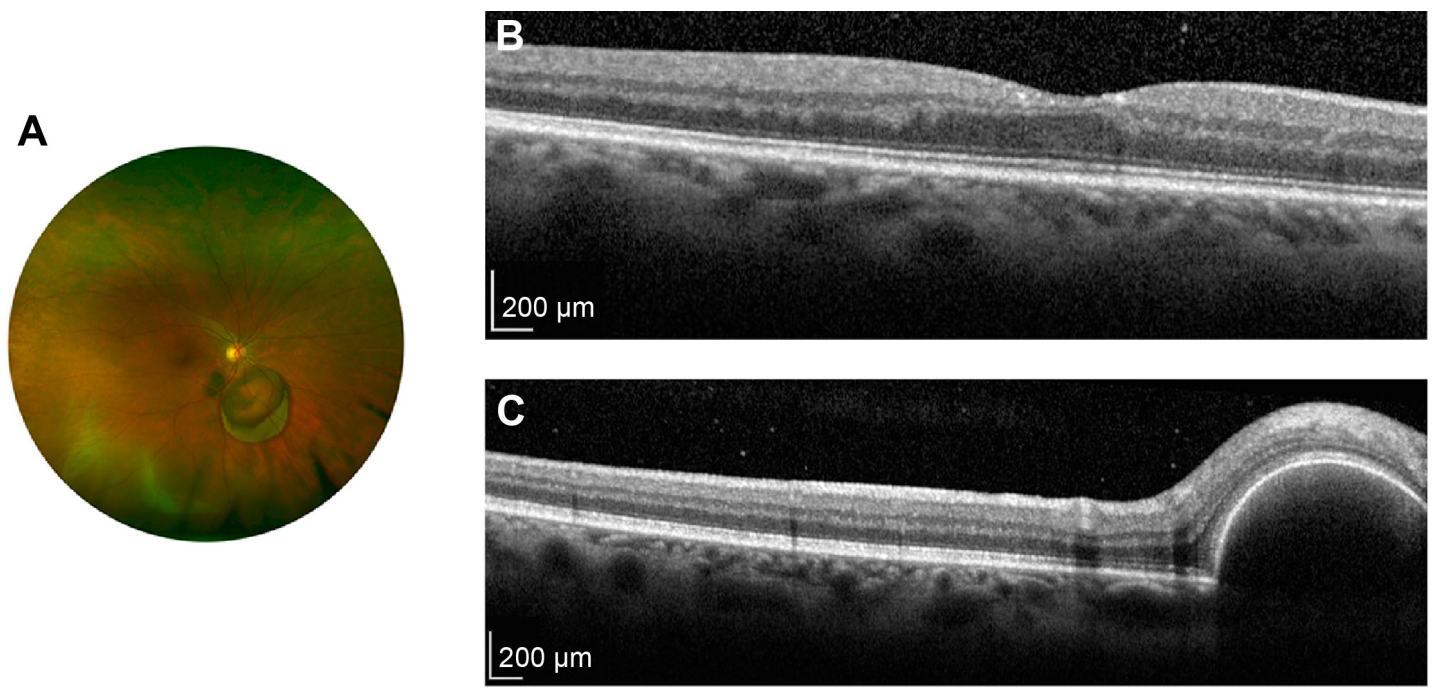

Figure 4 Case 4 ocular imaging.

Notes: Color fundus photograph OD of case 4 reveals a peripapillary sub-RPE hemorrhage and incidentally noted inferior retinal detachment (A). EDI-OCT OD of the macula (B) and of the retina adjacent to the hemorrhagic PED (C) reveals thick choroid with dilated outer choroidal vessels.

Abbreviations: OD, oculus dexter (right eye); RPE, retinal pigment epithelium; EDI-OCT, enhanced-depth imaging optical coherence tomography; PED, pigment epithelial detachment.
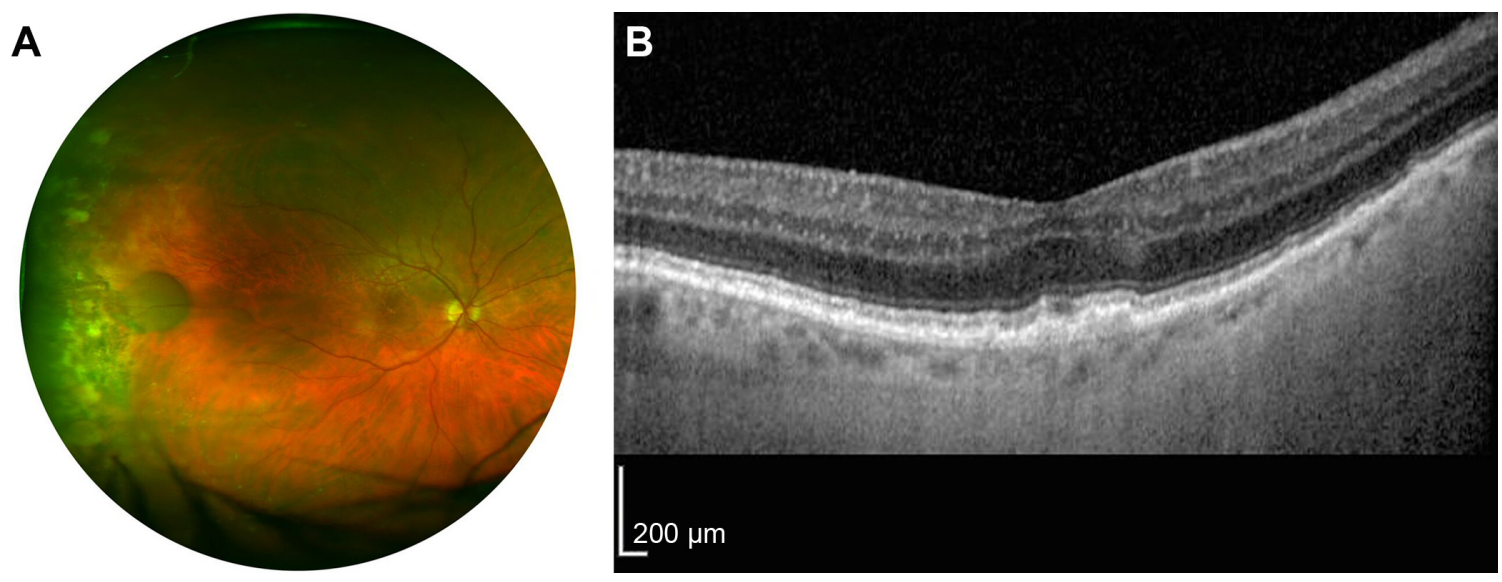

Figure 5 Case 5 ocular imaging.

Notes: Color fundus photograph OD of case 5 reveals drusen and RPE changes with a hemorrhagic lesion in the far temporal periphery (A). EDI-OCT of the macula OD reveals RPE changes and a thin choroid (B).

Abbreviations: OD, oculus dexter (right eye); RPE, retinal pigment epithelium; EDI-OCT, enhanced-depth imaging optical coherence tomography. 

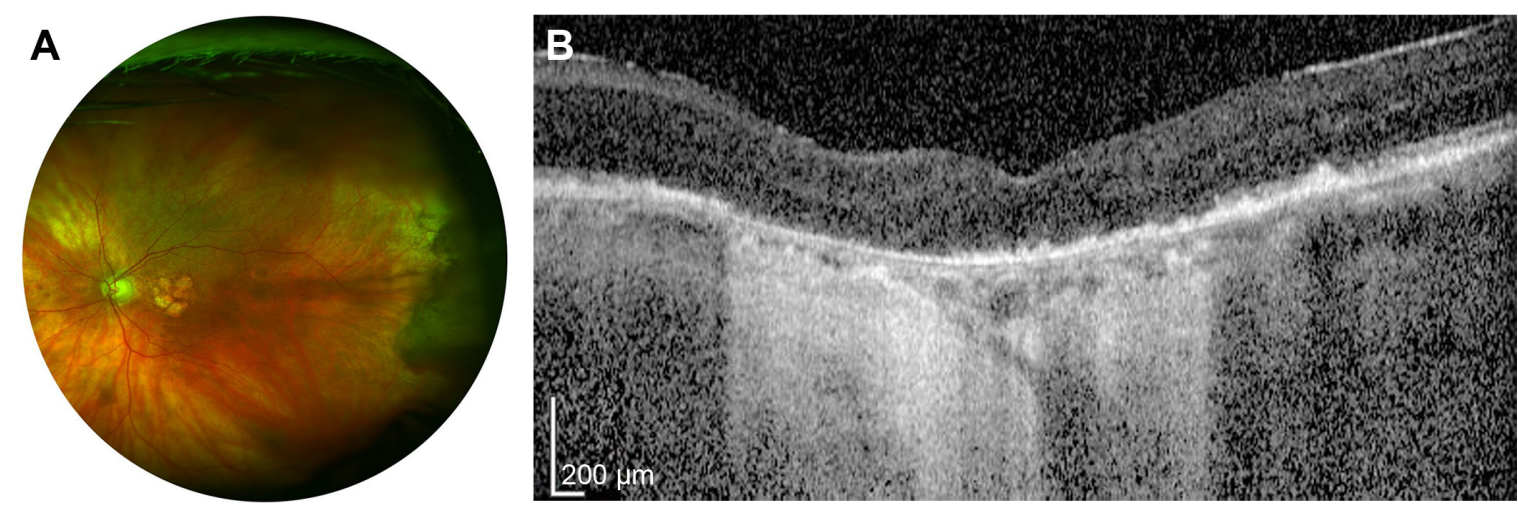

Figure 6 Case 6 ocular imaging.

Notes: Color fundus photograph OS of case 6 reveals drusen and RPE changes, as well as a far peripheral elevated lesion (A). EDI-OCT of the central macula OS reveals RPE changes with choroidal thinning (B).

Abbreviations: OS, oculus sinister (left eye); RPE, retinal pigment epithelium; EDI-OCT, enhanced-depth imaging optical coherence tomography.

revealed pachychoroidopathy. This suggests that even in cases where the extramacular CNVM itself is too peripheral for OCT imaging, choroidal features visible in the macula itself may support a diagnosis of pachychoroid neovasculopathy. This finding also begs the question of whether presence of such an extramacular CNVM in the setting of pachychoroidopathy in the macula as well suggests an underlying risk for future pigment epitheliopathy or CNVM risk in the macula itself. Further studies with more patients and more long-term follow-up are necessary.

Both cases of extramacular CNVMs without pachychoroid features also had age-related macular degeneration, and their macular choroids exhibited marked thinning, as is common in age-related macular degeneration. Interestingly, the extramacular CNVMs in both of these cases were also far more peripheral than those noted in the four cases with pachychoroidopathy.

Extramacular CNVMs (also known as peripheral disciform lesions, extramacular disciform, or peripheral exudative hemorrhagic chorioretinopathy) comprise a broad diagnostic category. It is now well known that many of these extramacular CNVMs are PCV lesions, ${ }^{13}$ although large studies evaluating extramacular CNVMs have demonstrated both the absence and presence of PCV-like polyps. ${ }^{13-15}$ Since PCV is implicated in some extramacular CNVMs and also falls within the pachychoroid spectrum, the pachychoroidopathy noted in cases 1-4 in this series may reflect the known relationship of PCV to extramacular CNVMs. Indeed, in the current study, only one patient received ICG angiography (case 2), and that patient exhibited polypoidal changes consistent with PCV. ICG was not performed or was unable to be performed due to the far peripheral location of the CNVMs in the other cases. The lack of ICG for the other cases is a major limitation of this study, as it is therefore unclear whether there were any cases of extramacular pachychoroidopathy in the absence of PCV features. If pachychoroidopathy were demonstrated in the absence of polyps on ICG in these lesions, pachychoroidopathy may offer an etiology for lesions previously considered idiopathic due to absence of polyps. Prior reports have demonstrated macular pachychoroid neovasculopathy in the absence of PCV ${ }^{1,3}$ Further studies of pachychoroid extramacular CNVMs with more extensive ICG evaluation are necessary.

In addition to providing a unifying theme for several related conditions, as well as potentially offering a diagnosis for lesions previously thought to be idiopathic, the conception of pachychoroidopathy may have diagnostic and therapeutic implications. A recent genetic study evaluating eleven AMD susceptibility genes, including complement factor $\mathrm{H}$ and ARMS2 rs 10490924 found that patients with pachychoroid neovasculopathy exhibited markedly less susceptibility than those with AMD. ${ }^{16}$ This suggests that a distinct pathogenesis may be implicated in these pachychoroid-driven diseases. Consistent with this finding, we noted that both cases of extramacular CNVM without pachychoroid features in the current study exhibited underlying AMD. Similarly, it is well known that several pachychoroid diseases, including chronic CSCR and PCV, may respond less to anti-VEGF agents and better to photodynamic therapy, than AMD. Thus, the distinction between neovascularization related to or unrelated to pachychoroidopathy may have important implications for therapeutics. Consistent with this, one patient in this series (case 1) with pachychoroid neovasculopathy was treated with anti-VEGF agents with no significant response in exam or symptoms; photodynamic therapy was offered, but the patient deferred. 
This study had several limitations. The number of reported cases is small. ICG was not performed or was unable to be performed (due to the far peripheral location of the lesions) in most of the cases evaluated; wide-field FA was used instead. As discussed above, ICG imaging to confirm absence or presence of polypoidal lesions would be instructive. In its absence, it is not possible to determine whether the pachychoroidopathy noted in these extramacular CNVMs reflects the known relation of these lesions to PCV or if there exists cases of extramacular pachychoroid neovasculopathy in the absence of PCV. Finally, the far peripheral location of the extramacular CNVMs in two cases, both of which were felt not to exhibit pachychoroidopathy, prevented the abilitiy to perform OCT through the lesion itself. In both of these cases, choroidal features in the macula were used as a surrogate and revealed thin choroid not consistent with pachychoroidopathy. If the pathology in these extramacular CNVMs is a focal choroidopathy localized to the region of the CNVM itself, using macular choroidal findings to inform the diagnosis of peripheral CNVMs may not be appropriate. However, we noted in this series that in all four cases where sub-CNVM OCTs were able to be performed (cases 1-4), the choroidal findings in the macula mirrored those in near the lesion itself.

\section{Disclosure}

The authors report no conflicts of interest in this work.

\section{References}

1. Pang CE, Freund KB. Pachychoroid neovasculopathy. Retina. 2014; 35(1):1-9.

2. Warrow DJ, Hoang QV, Freund KB. Pachychoroid pigment epitheliopathy. Retina. 2013;33(8):1659-1672.

3. Dansingani KK, Balaratnasingam C, Naysan J, Freund KB. En face imaging of pachychoroid spectrum disorders with swept-source optical coherence tomography. Retina. 2016;36(3):499-516.

Clinical Ophthalmology

\section{Publish your work in this journal}

Clinical Ophthalmology is an international, peer-reviewed journal covering all subspecialties within ophthalmology. Key topics include: Optometry; Visual science; Pharmacology and drug therapy in eye diseases; Basic Sciences; Primary and Secondary eye care; Patient Safety and Quality of Care Improvements. This journal is indexed on Submit your manuscript here: http://www.dovepress.com/clinical-ophthalmology-journal
4. Khan S, Engelbert M, Imamura Y, Freund KB. Polypoidal choroidal vasculopathy: simultaneous indocyanine green angiography and eye-tracked spectral domain optical coherence tomography findings. Retina. 2012;32(6):1057-1068.

5. Tsujikawa A, Ojima Y, Yamashiro K, et al. Development of polypoidal lesions in age-related macular degeneration. Eye. 2011;25(4): 481-488.

6. Tamura H, Tsujikawa A, Otani A, et al. Polypoidal choroidal vasculopathy appearing as classic choroidal neovascularization on fluorescein angiography. Br J Ophthalmol. 2007;91(9):1152-1159.

7. Freund KB, Zweifel SA, Engelbert M. Do we need a new classification for choroidal neovascularization in age-related macular degeneration? Retina. 2010;30(9):1333-1349.

8. Sasahara M, Tsujikawa A, Musashi K, et al. Polypoidal choroidal vasculopathy with choroidal vascular hyperpermeability. Am JOphthalmol. 2006;142(4):601-607.

9. Ahuja RM, Downes SM, Stanga PE, Koh AH, Vigerling JR, Bird AC. Polypoidal choroidal vasculopathy and central serous chorioretinopathy. Ophthalmology. 2001;108(6):1009-1010.

10. Yannuzzi LA, Freund KB, Goldbaum M, et al. Polypoidal choroidal vasculopathy masquerading as central serous chorioretinopathy. Ophthalmology. 2000;107(4):767-777.

11. Ueta $T$, Obata R, Inoue $Y$, et al. Background comparison of typical age-related macular degeneration and polypoidal choroidal vasculopathy in Japanese patients. Ophthalmology. 2009;116(12): 2400-2406.

12. Koizumi H, Yamagishi T, Yamazaki T, Kinoshita S. Relationship between clinical characteristics of polypoidal choroidal vasculopathy and choroidal vascular hyperpermeability. Am J Ophthalmol. 2013;155(2): 305-313.

13. Mantel I, Shalenbourg A, Zografos L. Peripheral exudative hemorrhagic chorioretinopathy: polypoidal choroidal vasculopathy and hemodynamic modifications. Am J Ophthalmol. 2012;153(5):910-922.

14. Mantel I, Uffer S, Zografos L. Peripheral exudative hemorrhagic chorioretinopathy: a clinical, angiographic, and histologic study. Am J Ophthalmol. 2009;148(6):932-938.

15. Goldman DR, Freund KB, McCannel CA, Sarraf D. Peripheral polypoidal choroidal vasculopathy as a cause of peripheral exudative hemorrhagic chorioretinopathy: a report of 10 cases. Retina. 2013;33(1): $48-55$.

16. Miyake M, Ooto S, Yamashiro K, et al. Pachychoroid neovasculopathy and age-related macular degeneration. Scientific Reports. 2015;5: $1-11$.

\section{Dovepress}

PubMed Central and CAS, and is the official journal of The Society of Clinical Ophthalmology (SCO). The manuscript management system is completely online and includes a very quick and fair peer-review system, which is all easy to use. Visit http://www.dovepress.com/ testimonials.php to read real quotes from published authors. 